\title{
COUNTING ADDITIVE DECOMPOSITIONS OF QUADRATIC RESIDUES IN FINITE FIELDS
}

\author{
SIMON R. BLACKBURN, SERGEI V. KONYAGIN, \\ AND IGOR E. SHPARLINSKI
}

\begin{abstract}
We say that a set $\mathcal{S}$ is additively decomposed into two sets $\mathcal{A}$ and $\mathcal{B}$ if $\mathcal{S}=\{a+b: a \in \mathcal{A}, b \in \mathcal{B}\}$. A. Sárközy has recently conjectured that the set $\mathcal{Q}$ of quadratic residues modulo a prime $p$ does not have nontrivial decompositions. Although various partial results towards this conjecture have been obtained, it is still open. Here we obtain a nontrivial upper bound on the number of such decompositions.
\end{abstract}

\section{INTRODUCTION}

Given two subsets $\mathcal{A}, \mathcal{B} \subseteq \mathbb{F}_{q}$ of the finite field $\mathbb{F}_{q}$ of $q$ elements, we define their sum as

$$
\mathcal{A}+\mathcal{B}=\{a+b: a \in \mathcal{A}, b \in \mathcal{B}\} .
$$

A set $\mathcal{S} \subseteq \mathbb{F}_{q}$ is called additively decomposable into two sets if $\mathcal{S}=\mathcal{A}+\mathcal{B}$ for some sets $\mathcal{A}, \mathcal{B}$ with

$$
\min \{\# \mathcal{A}, \# \mathcal{B}\} \geq 2 .
$$

Sárközy [6] has conjectured that the set $\mathcal{Q}$ of quadratic residues modulo a prime $p$ does not have additive decompositions and shown towards this conjecture that any additive decomposition

$$
\mathcal{Q}=\mathcal{A}+\mathcal{B}
$$

satisfies

$$
\frac{p^{1 / 2}}{3 \log p} \leq \min \{\# \mathcal{A}, \# \mathcal{B}\} \leq \max \{\# \mathcal{A}, \# \mathcal{B}\} \leq p^{1 / 2} \log p .
$$

The method also works for an arbitrary finite field of odd characteristic. In [8] this result has been improved to

$$
c q^{1 / 2} \leq \min \{\# \mathcal{A}, \# \mathcal{B}\} \leq \max \{\# \mathcal{A}, \# \mathcal{B}\} \leq C q^{1 / 2},
$$

2010 Mathematics Subject Classification. 11B13, 11L40.

Key words and phrases. Additive decompositions, finite fields, quadratic nonresidues character sums. 
for some absolute constants $C \geq c>0$ (and also generalised to other multiplicative subgroups of $\mathbb{F}_{q}^{*}$ ).

Shkredov 7] has recently made remarkable progress towards the conjecture of Sárközy [6] by showing that the conjecture holds with $\mathcal{A}=\mathcal{B}$. That is, $\mathcal{Q} \neq \mathcal{A}+\mathcal{A}$ for any set $\mathcal{A} \subseteq \mathbb{F}_{p}$.

Furthermore, Dartyge and Sárközy 1 have made a similar conjecture for the set $\mathcal{R}$ of primitive roots modulo $p$. We also refer to [1, 2, 6] for further references about set decompositions.

For an odd prime power $q$ we denote by $N(q)$ the total number of pairs $(\mathcal{A}, \mathcal{B})$ of sets $\mathcal{A}, \mathcal{B} \subseteq \mathbb{F}_{q}$ that provide an additive decomposition of the set of quadratic residues of $\mathbb{F}_{q}$, that is, the set $\mathcal{Q}=\left\{x^{2}: x \in\right.$ $\left.\mathbb{F}_{q}^{*}\right\}$. The conjecture of Sárközy [6] is equivalent to the statement that $N(q)=0$ when $q$ is an odd prime (and is probably true for any odd prime power as well).

The bound (1) implies

$$
N(q) \leq \exp \left(O\left(q^{1 / 2} \log q\right)\right) .
$$

Here we obtain a more precise estimate:

Theorem 1. For any odd prime power $q$, we have

$$
N(q) \leq \exp \left(O\left(q^{1 / 2}\right)\right) \text {. }
$$

Finally, we remark that the argument we use to prove Theorem 1 can be extended to prove results on additive decompositions of many other "multiplicatively" defined sets, such as cosets of multiplicative groups and sets of primitive elements of $\mathbb{F}_{q}^{*}$. See [1, 8] for analogues of (1) for such sets.

\section{Bounds of Multiplicative Character Sums}

As usual, we use the expressions $A \ll B$ and $A=O(B)$ to mean $|A| \leq c B$ for some constant $c$.

We recall the following bound on a double character sum due to Karatsuba [4, see also [5, Chapter VIII, Problem 9], which can easily be derived from the Weil bound (see [3. Corollary 11.24]) and the Hölder inequality.

Lemma 2. For any integer $\nu \geq 1$, sets $\mathcal{U}, \mathcal{V} \subseteq \mathbb{F}_{q}$ and nontrivial multiplicative character $\chi$ of $\mathbb{F}_{q}$, we have

$$
\sum_{u \in \mathcal{U}} \sum_{v \in \mathcal{V}} \chi(u+v) \ll(\# \mathcal{U})^{1-1 / 2 \nu} \# \mathcal{V} q^{1 / 4 \nu}+(\# \mathcal{U})^{1-1 / 2 \nu}(\# \mathcal{V})^{1 / 2} q^{1 / 2 \nu}
$$

where the implied constant depends only on $\nu$.

We obtain the following result as a corollary of Lemma 2 : 
Lemma 3. For any $\varepsilon>0$ if for two sets $\mathcal{U}, \mathcal{V} \subseteq \mathbb{F}_{q}$ with $\# \mathcal{V} \geq q^{\varepsilon}$ and a nontrivial multiplicative character $\chi$ of $\mathbb{F}_{q}$, we have $\chi(u+v)=1$ for all pairs $(u, v) \in \mathcal{U} \times \mathcal{V}$, then $\# \mathcal{U} \ll q^{1 / 2}$ where the implied constant depends only on $\varepsilon$.

Proof. We see from Lemma 2 that

$$
\begin{aligned}
\# \mathcal{U} \# \mathcal{V} & =\sum_{u \in \mathcal{U}} \sum_{v \in \mathcal{V}} \chi(u+v) \\
& \ll(\# \mathcal{U})^{1-1 / 2 \nu} \# \mathcal{V} q^{1 / 4 \nu}+(\# \mathcal{U})^{1-1 / 2 \nu}(\# \mathcal{V})^{1 / 2} q^{1 / 2 \nu}
\end{aligned}
$$

Taking $\nu$ sufficiently large so that the first term dominates (for example, taking $\nu=\left\lceil(2 \varepsilon)^{-1}\right\rceil$ so that $\left.\# \mathcal{V} \geq q^{1 / 2 \nu}\right)$ we find that

$$
\# \mathcal{U} \# \mathcal{V} \ll(\# \mathcal{U})^{1-1 / 2 \nu} \# \mathcal{V} q^{1 / 4 \nu}
$$

which implies the result.

We remark that the bounds (11) follow from Sárközy's result [6] and Lemma 3. To see this, note that the upper bound follows by taking $\chi$ to be the quadratic character in Lemma 3 , and taking $\mathcal{U}=\mathcal{A}$ and $\mathcal{V}=\mathcal{B}$ (and then $\mathcal{U}=\mathcal{B}$ and $\mathcal{V}=\mathcal{A}$ ). The lower bound now follows since $\# \mathcal{Q} \leq \# \mathcal{A} \# \mathcal{B}$.

\section{Proof of Theorem 1}

The proof of Theorem 1 is instant from the following result, which is of independent interest.

For positive integers $k$ and $m$, let $N(k, m, q)$ denote the number of pairs $(\mathcal{A}, \mathcal{B})$ of sets $\mathcal{A}, \mathcal{B} \subseteq \mathbb{F}_{q}$ with $\# \mathcal{A}=k, \mathcal{B}=m$ such that $\mathcal{Q}=\mathcal{A}+\mathcal{B}$.

To simplify formulas we extend the definition of binomial coefficients to all non-negative real numbers. More precisely, for a real $z \geq 0$ and an integer $n$ we set

$$
\left(\begin{array}{l}
z \\
n
\end{array}\right)=\left(\begin{array}{c}
\lfloor z\rfloor \\
n
\end{array}\right) .
$$

Lemma 4. For any fixed $\varepsilon>0$ there is a constant $c>0$ such that for all integers $k$ and $m$ with $q>k>q^{\varepsilon}$ and $q>m>q^{\varepsilon}$, we have

$$
N(k, m, q) \leq\left(\begin{array}{c}
c q^{1 / 2} \\
k
\end{array}\right)\left(\begin{array}{c}
c q^{1 / 2} \\
m
\end{array}\right) .
$$

Proof. We fix a set $\mathcal{V} \subseteq \mathbb{F}_{q}$ of size $\# \mathcal{V}=\left\lfloor q^{\varepsilon / 2}\right\rfloor$. We estimate the number $N(\mathcal{V}, k, m, q)$ of sets $\mathcal{A}, \mathcal{B} \subseteq \mathbb{F}_{q}$ with $\# \mathcal{A}=k, \mathcal{B}=m$ such that

$$
\mathcal{Q}=\mathcal{A}+\mathcal{B} \quad \text { and } \quad \mathcal{V} \subseteq \mathcal{B}
$$


Let $\chi$ be the quadratic character. Let $\mathcal{U}$ be the set of elements $u \in \mathbb{F}_{q}$ such that for every $v \in \mathcal{V}$ we have $\chi(u+v)=1$. We see from Lemma 3 that $\# \mathcal{U} \ll q^{1 / 2}$.

Any set $\mathcal{A}$ which contributes to $N(\mathcal{V}, k, m, q)$ satisfies $\mathcal{A} \subseteq \mathcal{U}$. Hence there are at most

$$
\left(\begin{array}{c}
\# \mathcal{U} \\
k
\end{array}\right) \leq\left(\begin{array}{c}
c_{1} q^{1 / 2} \\
k
\end{array}\right)
$$

possibilities for $\mathcal{A}$ (where $c_{1}>0$ is some constant that depends only on $\varepsilon)$.

Suppose now that $\mathcal{A}$ is chosen. Fixing an arbitrary set of $\left\lfloor q^{\varepsilon / 2}\right\rfloor$ elements of $\mathcal{A}$ and using the same argument we see that that the remaining elements of $\mathcal{B}$ always belong to some fixed set $\mathcal{W} \subseteq \mathbb{F}_{q}$ of size $\# \mathcal{W} \ll q^{1 / 2}$. Therefore, there are at most

$$
\left(\begin{array}{c}
\# \mathcal{W} \\
k
\end{array}\right) \leq\left(\begin{array}{c}
c_{2} q^{1 / 2} \\
m
\end{array}\right)
$$

possibilities for the remaining elements of $\mathcal{B}$ (where $c_{2}>0$ is some constant that depends only on $\varepsilon$ ). Hence, combining (22) and (31), we obtain

$$
N(\mathcal{V}, k, m, q) \leq\left(\begin{array}{c}
c_{1} q^{1 / 2} \\
k
\end{array}\right)\left(\begin{array}{c}
c_{2} q^{1 / 2} \\
m
\end{array}\right)
$$

Summing over all choices for $\mathcal{V}$ yields

$$
\begin{aligned}
N(k, m, q) & \leq\left(\begin{array}{c}
q \\
q^{\varepsilon / 2}
\end{array}\right)\left(\begin{array}{c}
c_{1} q^{1 / 2} \\
k
\end{array}\right)\left(\begin{array}{c}
c_{2} q^{1 / 2} \\
m
\end{array}\right) \\
& \leq q^{q^{\varepsilon / 2}}\left(\begin{array}{c}
c_{1} q^{1 / 2} \\
k
\end{array}\right)\left(\begin{array}{c}
c_{2} q^{1 / 2} \\
m
\end{array}\right)
\end{aligned}
$$

which concludes the proof.

Now, using the fact that $N(k, m, q) \neq 0$ only if $q^{1 / 2} \ll k \ll q^{1 / 2}$ and $q^{1 / 2} \ll m \ll q^{1 / 2}$, see (1), we easily derive Theorem 1 from Lemma 4 .

\section{ACKNOWLEDGMENTS}

During the preparation of the work, the second author was supported by Russian Fund for Basic Research, Grant N. 14-01-00332, and Program Supporting Leading Scientific Schools, Grant Nsh-3082.2014.1; the third author was supported by the Australian Research Council, Grant DP140100118. 


\section{REFERENCES}

[1] C. Dartyge and A. Sárközy, 'On additive decompositions of the set of primitive roots modulo p', Monat. Math., 169 (2013), 317-328.

[2] C. Elsholtz, 'A survey on additive and multiplicative decompositions of sumsets and of shifted sets', Combinatorial Number Theory and Additive Group Theory, Birkhäuser, 2009, 213-231.

[3] H. Iwaniec and E. Kowalski, Analytic number theory, Amer. Math. Soc., Providence, RI, 2004.

[4] A. A. Karatsuba, 'The distribution of values of Dirichlet characters on additive sequences', Doklady Acad. Sci. USSR, 319 (1991), 543-545 (in Russian).

[5] A. A. Karatsuba, Basic analytic number theory, Springer-Verlag, 1993.

[6] A. Sárközy, 'On additive decompositions of the set of quadratic residues modulo p', Acta Arith., 155 (2012), 41-51.

[7] I. D. Shkredov, 'Sumsets in quadratic residues', Acta Arithmetica, (to appear).

[8] I. E. Shparlinski, 'Additive decompositions of subgroups of finite fields', SIAM J. Discr. Math., 27 (2013), 1870-1879.

Department of Mathematics, Royal Holloway University of LonDon, Egham, Surrey, TW20 0EX, UK

E-mail address: s.blackburn@rhul.ac.uk

Steklov Mathematical Institute, 8, Gubkin Street, Moscow, 119991, RUssia

E-mail address: konyagin@mi.ras.ru

Department of Pure Mathematics, University of New South Wales, SYDNEY, NSW 2052 AUSTRALIA

E-mail address: igor.shparlinski@unsw.edu.au 